\title{
¿Un espacio para pensar alternativas? La academia latinoamericana de derecho internacional económico frente al orden económico global $^{* *}$
}

\author{
A space to imagine alternatives? \\ The Latin American scholarship on \\ international economic law and the \\ formation of the global economic order
}

SUMARIO

Introducción. I La labor del derecho internacional económico: "ordenar" las relaciones económicas internacionales. a. Ordenando el mundo creamos un mundo. b. La indeterminación de las reglas, las racionalidades del derecho internacional económico y el problema del orden. II. El derecho internacional y sus principales racionalidades: reconocimiento político y económico en Latinoamérica. a. La aspiración de ser parte de los países civilizados: igualdad y soberanía. b. La aspiración de ser parte del mundo desarrollado: igualdad, desarrollo y rule of law. III. La academia latinoamericana de DIE y su contribución al orden económico global. a. La contribución a través de la enseñanza. b. La contribución a través de la defensa de los intereses del Estado. c. La contribución a través de la investigación. Conclusiones.

RESUMEN

Esta contribución tiene por objetivo analizar el rol de la academia latinoamericana de derecho internacional económico frente al orden económico

* PhD, London School of Economics and Political Science. Docente investigador, Universidad Externado de Colombia. Agradezco los comentarios a todo o parte de este documento recibidos de José Manuel Álvarez, Carlos Correa, un revisor anónimo, y de los asistentes al II Congreso de la Red Colombiana de Derecho Internacional (Medellín, mayo de 2015) y al III Congreso Bianual de la Red Latinoamericana de Derecho Internacional Económico (Porto Alegre, octubre de 2015). Cualquier error u omisión es de mi exclusiva responsabilidad. Contacto: nicolas.perrone@uexternado.edu.co

** Recibido el 18 de enero de 2016, aprobado el 16 de mayo de 2016.

Para citar el artículo: N. M. PERRONE, ¿Un espacio para pensar alternativas? La academia latinoamericana de derecho internacional económico frente al orden económico global, Derecho del Estado n. ${ }^{\circ}$ 36, Universidad Externado de Colombia, enero-junio de 2016, pp. 199-226. DoI: http://dx.doi.org/10.18601/01229893.n36.07. 
global. Gran parte de los problemas que hoy sufren los latinoamericanos se vincula a la globalización económica. Condiciones laborales, acceso a medicamentos, uso de recursos naturales, entre otros, están íntimamente ligados al orden económico global. En el debate sobre estos problemas, no obstante, pocas veces se reconoce el rol fundamental que tiene la academia de derecho internacional económico. Si bien es cierto que el conocimiento articulado por la academia no es suficiente para explicar por qué los Estados actúan de cierta forma, quizás sea suficiente para entender por qué no actúan de otra manera. En este marco, el argumento central de esta contribución es que la academia de derecho internacional económico tiene un rol clave en la creación y reproducción del actual orden económico global. Si esto es correcto, la academia regional puede hacer más por Latinoamérica que servir de apoyo a las necesidades estatales, tanto de asesoría como de defensa de sus intereses en controversias legales. Al tomar conciencia de su rol constitutivo del orden económico global, la academia regional puede ser un medio fundamental para articular alternativas que permitan a Latinoamérica y su población encontrar su lugar en la globalización.

PALABRAS CLAVE

Derecho internacional económico, Latinoamérica, globalización, inversiones extranjeras, comercio internacional.

\section{ABSTRACT}

This article aims to analyse the role of the Latin American scholarship on international economic law in the formation of the global economic order. A large number of the problems that many Latin Americans face today relate to the global economy. Labour conditions, access to medicines, the use of natural resources, among others, are closely connected to the global economic order. In few instances, however, the debate on these problems recognises the fundamental role of the international economic law scholarship. Although the actual knowledge created by this scholarship does not explain why States behave in a certain way, it may serve to explain why they do not behave otherwise. This article argues that the scholarship on international economic law plays a crucial role in the creation and reproduction of the current global economic order. If this is correct, the regional scholarship can do more for Latin American than meeting the advisory and litigation needs of states. It can promote alternatives that can help the region and its people to find their place in the global economy. 
KEYWORDS

International Economic Law, Latin America, Globalisation, Foreign Investment, International Trade.

\section{INTRODUCCIÓN}

La economía global es arena de muchas luchas que tienen en vilo a distintas regiones y personas, entre ellas, a Latinoamérica y su gente. En esta arena están en juego los intereses de campesinos y comunidades aborígenes, y también de trabajadores, enfermos, y pequeños y medianos empresarios. Frente a estas tensiones, el derecho internacional económico (DIE) se presenta al mundo y a los latinoamericanos como el medio para regular conflictos comerciales y de inversiones entre Estados e inversores extranjeros. A poco que se la analice, no obstante, esta descripción no parece acorde con las tensiones antes señaladas. No tiene en cuenta que el DIE se ha transformado en el principal derecho de la globalización, y como tal es árbitro de los intereses y derechos tanto de las grandes empresas multinacionales como de casi todas las personas ${ }^{1}$. Esto no significa que el DIE tenga primacía formal sobre los derechos humanos o el derecho al medio ambiente. Pero, muchas veces, estos otros derechos internacionales juegan papeles algo secundarios frente a las reglas de derecho del comercio y las inversiones internacionales.

La creciente importancia del DIE justifica estudiar con detenimiento y especial interés no sólo sus reglas jurídicas, sino también la creación de conocimiento en DIE y el rol de la academia en esa creación. El rol de la academia puede parecer más bien modesto si aceptamos que su tarea es manejar el conjunto de reglas, determinadas o determinables, que gobiernan las relaciones económicas internacionales. Desde esta perspectiva, el DIE operaría bajo reglas relativamente claras que, a su vez, permitirían conocer fácilmente los intereses de cada Estado y cómo estos pueden maximizarlos. En términos comerciales, estos consistirían en ampliar sus mercados cediendo la menor cuota de mercado doméstico posible; en términos de inversiones extranjeras, en atraer toda la inversión extranjera posible cediendo la menor porción de soberanía ${ }^{2}$.

Lo que esta visión -probablemente dominante- no tiene en cuenta es que el DIE no es sólo el resultado de las negociaciones entre Estados. No es sólo el producto de un cúmulo de tratados internacionales. El conocimiento

1 En 1963, BAADE ya enfatizaba la importancia del DIE para el derecho laboral y los derechos de los trabajadores. Ver BAADE, H. Teaching International Economic Law. 16 Journal of Legal Education 59, 1963, 61-63.

2 Este tipo de análisis responde a visiones realistas o neoutilitarias de las relaciones internacionales. Ver RugGIE, J., What Makes the World Hang Together? Neo-utilitarianism and the Social Constructivist Challenge. 52: 4 International Organization 855, 1998. 
creado por la academia de DIE juega un papel central en la configuración del orden económico global. Este define no sólo la interpretación sino también las fuentes del derecho que dan sentido a, por ejemplo, el término 'trato justo y equitativo' en los tratados de protección de inversiones ${ }^{3}$. La arena donde operan las reglas de derecho es una forma de conocimiento creada por la propia academia de DIE $^{4}$. Este conocimiento da vida a los tratados firmados por todos los Estados, incluidos los latinoamericanos, y disciplina la negociación de cualquier obligación internacional en materia económica.

Pero el hecho de que el DIE sea parte del derecho internacional no significa que el conocimiento en esta materia sea creado sobre la base de una participación amplia y equitativa. En realidad, la creación de conocimiento en derecho internacional general tampoco respondería a esta caracterización. Siguiendo a Chimni, por ejemplo, se puede argumentar que el DIE es creado desde un espacio global ocupado por los intereses de una clase capitalista transnacional ${ }^{5}$. Si bien es difícil vincular a estos intereses con espacios geográficos concretos, gran parte del DIE enseñado en las universidades del mundo proviene del Norte global, y esto da a esos académicos un gran protagonismo en la creación de conocimiento de DIE. La academia latinoamericana de DIE indudablemente forma parte de la academia global. El DIE, como veremos, se presenta hoy al mundo como una disciplina global. En la práctica, sin embargo, los académicos regionales juegan un papel algo secundario en la creación de conocimiento en DIE, aunque no así en la diseminación o aplicación de ese conocimiento para resolver disputas concretas.

En este marco, la propuesta de este trabajo no es estudiar las reglas de DIE, sino la creación de conocimiento alrededor de esta área del derecho internacional, poniendo especial atención en el rol de la academia latinoamericana. A lo largo de este trabajo, por ello, me voy a referir tanto a reglas como a racionalidades. Este término, que voy a utilizar en el sentido weberiano de racionalidad instrumental, se refiere a la forma del conocimiento de DIE. Se trata de un conocimiento que promueve ciertos fines, por ejemplo el desarrollo económico, y determina que ciertos medios son necesarios para alcanzar esos fines ${ }^{6}$. Es importante mencionar aquí que si bien Weber reconoce formas plurales de racionalidad, también destaca que sólo son aceptadas como objetivas

3 Ver Paparinskis, M. The International Minimum Standard and Fair and Equitable Treatment. Oxford: Oxford University Press, 2013.

4 Simpson, G. On the Magic Mountain: Teaching Public International Law. 10: 1 European Journal of International Law 70, 1999, 85; RAsulov, A. The Structure of the International Legal Discourse. European Society of International Law, Florence Founding Conference, 2004, 1.

5 Ver Chimni, B. International institutions today: An imperial global State in the making. 15: 1 European Journal of International Law 1, 2004.

6 WeBER, M. El sentido de la 'neutralidad valorativa' de las ciencias sociológicas y económicas. En Ensayos sobre metodología sociológica. Buenos Aires: Amorrortu, 1973, 222-268. 
aquellas racionalidades reconocidas por un conocimiento técnico ${ }^{7}$. En el caso del DIE, se trata de un conocimiento vinculado al capitalismo económico que exige, por sobre todas las cosas, asegurar la posibilidad de calcular ${ }^{8}$.

Según Ruggie, estas racionalidades motivan y justifican el accionar de los Estados. Explican su accionar más allá de la obligatoriedad de una regla o la posible utilidad en su cumplimiento ${ }^{9}$. Si bien las racionalidades no sirven para explicar causalmente las acciones de los Estados, no proveen una visión determinista del mundo, éstas sirven para explicar por qué los Estados no actúan de otra forma ${ }^{10}$. Así, la construcción de racionalidades es una herramienta fundamental para mantener el orden en las relaciones económicas internacionales. Estas sirven para definir los intereses de los jugadores, determinando sus conductas a través de incentivos que, en última instancia, afectan su subjetividad ${ }^{11}$.

El argumento central de esta contribución es que la academia de DIE cumple un rol central como generadora de conocimiento en esta materia, especialmente, de sus racionalidades. Esto quiere decir que la academia regional que se ocupa del DIE podría tener un rol más importante que la simple reproducción y aplicación de reglas de derecho. Podría ser, también, un medio para conocer y modificar las racionalidades del DIE, creando nuevas oportunidades para la región.

La primera parte de este trabajo analiza la visión que tiene la academia de derecho internacional de sí misma, especialmente la academia de DIE, y de qué forma los académicos en derecho internacional contribuyen al orden económico global. La segunda parte estudia las racionalidades que emergen del DIE desde una perspectiva latinoamericana, haciendo hincapié en su rol constitutivo del orden económico global. La última parte toma estos elementos para analizar en detalle la participación de la academia latinoamericana en la difusión y consolidación del orden económico tanto regional como global. La conclusión es que los intereses en juego en el ámbito del DIE son demasiado importantes para que la academia regional sólo tenga un rol pasivo frente a la creación de conocimiento en esta materia. Cualquiera sea la opinión que se tenga sobre el orden económico global, sea a favor o en contra de la mayor participación del Estado en la vida económica, es necesario saber de qué forma se consolida este orden y cuáles son los márgenes de maniobra y posibles

7 Ibíd.

8 Weber, M. Parliament and Government in Germany under a New Political Order. En Lassman, P. (ed.), Weber: Political Writings. Cambridge: Cambridge University Press, 1994, 147-148.

9 RUGGIE, supra nota 2, 859-860.

10 Ibíd., 869.

11 Ibíd., 862-864. Foucault, M., Technologies of the Self. En Rabinov, P. (ed.), Ethics: Subjectivity and Truth. New York: The New York Press, 1997, 223-251. 
estrategias de cambio. De otra forma, como advierte Aldo Ferrer, Latinoamérica no podrá "decidir [su] propio destino dentro de la globalización" 12 .

\section{LA LABOR DEL DERECHO INTERNACIONAL ECONÓMICO: "ORDENAR" LAS RELACIONES ECONÓMICAS INTERNACIONALES}

\section{a. Ordenando el mundo creamos un mundo}

Los académicos ubicados en el mainstream de derecho internacional suelen ver la razón de ser del derecho internacional, aquello que da sentido a su profesión, en su labor de ordenar las relaciones internacionales ${ }^{13}$. Inspirados en el ideal de KANT de la paz perpetua, tienen por misión regular las imperfectas relaciones internacionales, a fines de evitar ante todo la guerra.

No es muy distinto en el caso del DIE. El mainstream de la academia sostiene que es necesario regular las relaciones económicas internacionales. En el peor de los casos, estas relaciones también pueden llevar a la guerra. El férreo proteccionismo de la década de 1930 es indicado por muchos como uno de los detonantes de la Segunda Guerra Mundial ${ }^{14}$. La paz, además, es fundamental para el desarrollo eficiente de la actividad económica internacional. La solución pacífica de controversias es un pilar fundamental del orden económico internacional, siendo necesaria para promover la liberalización progresiva del comercio, las inversiones extranjeras y el desarrollo económico ${ }^{15}$.

Siguiendo esta línea, el mainstream de la academia en derecho internacional se caracteriza por asumir que ya existen las relaciones internacionales entre Estados, tanto políticas como económicas. Estas relaciones, incluso, preexistirían al derecho internacional. Sin embargo, estos académicos consideran necesario regular estas relaciones o al menos crear los mecanismos adecuados para que los Estados puedan autorregularse. Esta visión domina a los académicos neorrealistas y liberales neoinstitucionalistas.

RUGGIE critica estas posturas, no obstante, porque si bien hay reglas que regulan, también hay reglas que constituyen relaciones ${ }^{16}$. En la práctica, quizás se trata de categorías ideales y no es posible encontrar reglas totalmente reguladoras o totalmente constitutivas. Pero el DIE, como el derecho

12 FERRER, A. La importancia de las ideas propias sobre el desarrollo y la globalización. 173: 44 Revista Problemas del Desarrollo 163, 2013, 166.

13 Rasulov, A. supra n. 4, p. 2.

14 La guerra de aranceles comenzó con el dictado de la Smoot-Hawley Tariff Act en Estados Unidos. Trebilcock, M. y Howse, R. The regulation of international trade. New York: Routledge, 1999, 19.

15 Petersmann, E.U. Transformation of the World Trading System through the 1994 Agreement Establishing the World Trade Organization. 6 European Journal of International Law 161, 1995.

16 RugGie, J. supra n 2, 871. Ver, también, LANG, A. World trade law after neoliberalism: re-imagining the global economic order. Oxford: Oxford University Press, 2011, 160 ss. 
internacional, no sólo ordena las relaciones internacionales sino que también las constituye, dándoles formas particulares e imprimiéndoles ciertas dinámicas. Las relaciones entre los Estados pueden ser algo natural, partiendo de la premisa de que los seres humanos son políticos por naturaleza. Pero sea esto cierto o no, es indiscutible que los términos de las relaciones entre Estados, por ejemplo según su carácter civilizado o incivilizado, no son algo natural sino creado por el derecho internacional. Otro tanto podría decirse de las relaciones en términos de Estados desarrollados y en desarrollo. Estas categorías dan forma a lo que hay que regular. Hay Estados que deben tomar ciertas medidas para desarrollarse; otros, en cambio, se presentan al mundo como desarrollados. Las corrientes críticas del derecho internacional han enfatizado el rol de estas racionalidades por décadas ${ }^{17}$.

A pesar de esto, el mainstream del DIE opera bajo la premisa de que este no tiene un rol fundamental en la constitución de las relaciones económicas entre los Estados ${ }^{18}$. Desde esta visión formalista, el DIE se presenta como una serie de reglas que se aplican universalmente, basadas en los principios de igualdad, soberanía y pacta sunt servanda ${ }^{19}$. Estas reglas serían objetivas e independientes de los intereses particulares de cualquiera de los Estados, dando una impresión de igualdad entre todos los actores de la comunidad internacional. Esta igualdad se manifiesta, por ejemplo, en el principio de reciprocidad en las negociaciones comerciales, lo que equivale a decir que toda concesión comercial requiere una contraprestación. Así, escuchamos sistemáticamente que los tratados económicos son actos de cooperación y coordinación, entre dos o más Estados, que buscan alcanzar objetivos comunes ${ }^{20}$.

Un ejemplo de esta tensión entre regulación y constitución se encuentra en el derecho internacional de inversiones. Este régimen busca proteger a los inversores extranjeros del maltrato que puedan darles los Estados luego de establecer sus inversiones. No obstante, lo que ocurre antes del establecimiento y los posteriores derechos de los inversores son temas ignorados casi por completo. Durante el período anterior a la inversión extranjera se aplica casi sin limitaciones la autonomía de las partes, como si se tratara de actores en igualdad de condiciones, cuando en realidad las grandes empresas multinacionales casi siempre deciden dónde y en qué condiciones invertir.

17 Entre otros, cabe destacar el trabajo de ANGHIE: ver ANGHIE, A. Imperialism, Sovereignty and the Making of International Law. New York: Cambridge University Press, 2004.

18 La principal excepción se encuentra, probablemente, en la literatura del período que va desde 1950 a 1970 , es decir, durante el auge de la teoría de la dependencia y del derecho al desarrollo. Ver, entre otros, BECKER LoRCA, A. International Law in Latin America or Latin American International Law-Rise, Fall, and Retrieval of a Tradition of Legal Thinking and Political Imagination. 47 Harvard International Law Journal 283 (2006), 296-7.

19 KoskeNNIEMI, M. The Politics of International Law. 1 European Journal of International Law 4 (1990).

20 Ver, p. еj., Аввотт, K. у SNIDAL, D. Why states act through formal international organizations. 42:1 Journal of conflict resolution 3 (1998). 
Luego del establecimiento, cuando el Estado se volvería más fuerte gracias a su poder soberano, sí es necesaria la intervención del DIE para regular la relación entre el "débil" inversor extranjero y el "fuerte" Estado ${ }^{21}$.

El problema de sobrevalorar la visión reguladora del DIE es que se deja de percibir muchas consecuencias de esta área del derecho internacional. Por un lado, bajo la influencia del derecho al desarrollo, autores latinoamericanos ya criticaron -durante las décadas de 1960 y 1970 - una visión formal del principio de igualdad ${ }^{22}$. Por otro lado, el DIE tiene efectos sobre las personas y las comunidades. Si existían dudas, las transformaciones profundas de la década de 1990 las han despejado. El DIE se ha inmiscuido en la vida de cada quien, y aún así son pocos los que estudian el rol del DIE en nuestra vida diaria.

\section{b. La indeterminación de las reglas, las racionalidades del derecho internacional económico y el problema del orden}

Las reglas que conforman el DIE son indeterminadas, como cualquier regla jurídica, pero esto no quiere decir que cada Estado pueda hacer lo que quiera. La idea de desarrollo es el ejemplo más claro: ¿qué es el desarrollo?, ¿cómo se alcanza? La indeterminación de la idea de desarrollo pone en riesgo el orden económico internacional. Si cada Estado pudiera hacer lo que quiera para desarrollarse, un objetivo que incluso puede tener significados muy distintos en distintas partes del mundo, las posibilidades de conflicto serían muy altas. Para ordenar las relaciones económicas satisfactoriamente, el DIE requiere de mecanismos que minimicen ese espacio de indeterminación ${ }^{23}$.

Durante el apogeo de la teoría de la dependencia, la idea de desarrollo fue utilizada por parte de la academia vinculada al DIE -incluida la latinoamericana- como una herramienta emancipadora respecto del orden existente ${ }^{24}$. Hoy, no obstante, esta idea se presenta principalmente como una disciplina que los países llamados desarrollados imponen a los que están algo menos desarrollados ${ }^{25}$. No por casualidad, mientras la idea de desarrollo servía tanto para emancipar como para disciplinar el accionar estatal en las décadas de 1960 y 1970, el orden económico global era más poroso e indeterminado. El régimen actual de inversiones, con sus más de tres mil tratados, es justificado

21 Ver Perrone, N. The International Investment Regime and Foreign Investors' Rights: Another View of a Popular Story. PhD Thesis, London School of Economics and Political Science, 2013, 107-20.

22 Gross Espiell, H. Derecho internacional del desarrollo. En Estudios de Derecho Eco-

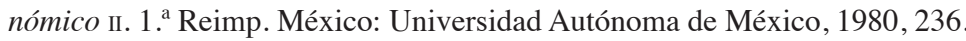

23 Rasulov, A. supra n. 4.

24 Ver BedjaOU, M. Towards a new international economic order. New York : Holmes \& Meier, 1979. En el caso latinoamericano, ver Gross Espiell, H. supra n. 22.

25 ANGHIE, supra n. 17; PAHUJA, S. Decolonising International Law: development, economic growth, and the politics of universality. Cambridge: Cambridge University Press, 2011, 132-5, 160-9. 
precisamente por la incertidumbre que prevalecía en esos años. Más allá de los tratados firmados, la consolidación de la interpretación dominante en los arbitrajes de inversiones requirió también cierto conocimiento de DIE. Un paso muy importante en esa dirección fue el trabajo de Paulsson sobre arbitration without privity ${ }^{26}$.

Los encargados de que las reglas indeterminadas del DIE tengan en la práctica un espacio reducido de indeterminación son los académicos, los expertos y quienes utilizan estas reglas en casos concretos. Esto se percibe hoy en la interpretación que hacen los árbitros de inversiones del trato justo y equitativo, por la que sostienen de manera mayoritaria que este estándar es casi el mismo en todos los tratados, a pesar de que las cláusulas son diferentes $^{27}$. Algo similar ocurre en el derecho de la omc. El término 'subsidio' puede tener distintas interpretaciones según la doctrina económica, pero en la OMC se ha alcanzado un consenso sobre su significado ${ }^{28}$. Tanto en el derecho de las inversiones extranjeras como en el derecho de la OMC, una clave para entender la interpretación dominante radica en el consenso sobre los fines de estos órdenes jurídicos: sea proteger la inversión extranjera o promover la liberalización progresiva del comercio.

Estos consensos son necesarios para el mantenimiento del orden económico global. Sin embargo, no son el resultado de negociaciones entre Estados, ni del ejercicio del poder material de los Estados más poderosos. Es aquí donde la academia de DIE juega un papel importante en la creación y articulación de conocimiento. En última instancia, es cierto, cuestiones como el significado del término 'subsidio' son definidas por los órganos jurisdiccionales del DIE. Pero estas decisiones son delimitadas por el trabajo de los académicos. El conocimiento en esta materia juega un papel importante en la aceptación o el rechazo de cualquier interpretación del trato justo y equitativo o del término 'subsidio'.

El mantenimiento del orden económico global, por otro lado, requiere más que resolver los posibles espacios de indeterminación. Un orden entre iguales puede resultar inestable y, por eso, las racionalidades creadas por la academia de DIE tienden a promover jerarquías -y no necesariamente igualdad-entre los Estados. Estas jerarquías son necesarias para que el DIE pueda mantener el orden en las relaciones económicas internacionales. Siempre que haya ganadores y perdedores, fuera y dentro de cada Estado, los primeros se ocupan de reproducir el orden. Proteger la inversión extranjera y promover la liberalización del comercio son racionalidades adecuadas ya que darían

26 Paulsson, J. Arbitration without privity. 10:2 ICSID Review, 1995, 232.

27 Ver PAPARINSKIS, supra n. 3.

28 Ver LANG, A. Governing 'As If': Global Subsidies Regulation and the Benchmark Problem. 67.1 Current Legal Problems, 2014, 135. 
como resultado, como señalan CHANG y WADE, ganadores y perdedores ${ }^{29}$. La igualdad, en cambio, pone a quienes se consideran perdedores en posición de intentar cambiar el orden vigente. Como explican STIGLITZ y RodRIK, los Estados en desarrollo necesitan de un espacio para experimentar ${ }^{30}$, pero en un mundo en constante experimentación es razonable asumir también un creciente desorden global.

La función del conocimiento dominante, entonces, no es sólo establecer racionalidades que delimitan el espacio de indeterminación, sino también otorgar legitimidad a las jerarquías económicas, ocultando la historia, en palabras de FouCAUlT ${ }^{31}$. Así, el conocimiento dominante debe ser capaz de mostrar a los socios de un tratado de libre comercio o de un acuerdo de protección de inversiones extranjeras como iguales, aunque no lo sean.

\section{EL DERECHO INTERNACIONAL Y SUS PRINCIPALES RACIONALIDADES: RECONOCIMIENTO POLÍTICO Y ECONÓMICO EN LATINOAMÉRICA}

A comienzos del siglo XIX, la comunidad latinoamericana se sumó a las relaciones internacionales como Estados independientes. Para ese momento ya existía un orden jurídico que daba forma a estas relaciones. Por un lado, existían reglas puntuales que regulaban la membrecía a la comunidad internacional de Estados. Por otro lado, había una serie de reglas generales que gobernaban las relaciones entre los miembros de la comunidad internacional, incluida su membrecía. Las reglas generales más importantes eran -y posiblemente siguen siendo- la igualdad y la soberanía de los Estados.

La igualdad de los Estados es la principal arena de las relaciones internacionales, pero sólo un análisis de cómo funciona esta igualdad en la práctica nos brinda una visión de la forma en que el derecho internacional no sólo regula sino también constituye estas relaciones. Una primera aproximación a la igualdad entre Estados nos muestra que se trata de una igualdad entre iguales, y no de una igualdad entre soberanos. Aquellos que se reconocen a sí mismos como soberanos son soberanos siempre que sean respetuosos de ciertas racionalidades económicas e institucionales. Es la persecución de esas racionalidades, justamente, lo que los vuelve iguales al resto de los Estados,

29 CHANG, H. J. Kicking away the ladder: development strategy in historical perspective. London: Anthem, 2002; WADE, R. What strategies are viable for developing countries today? The World Trade Organization and the shrinking of 'development space'. 10:4 Review of International Political Economy, 2003, 621.

30 RoDriK, D. The Global Governance of Trade - As If Development Really Mattered, United Nations Development Programme Background Paper, 2001; SERRA, N.; SPIEGEL, S. y STIGLiTZ, J. Introduction: From the Washington Consensus Towards a New Global Governance. En The Washington Consensus Reconsidered: Towards a New Global Governance. New York: Oxford University Press, 2008, 3-13.

31 Foucault, M. Society must be defended: Lectures at the Collège de France, 1975-76. London: Penguin, 2004, 98-126. 
y no a los otros: los incivilizados, los no soberanos. Como veremos, esta distinción entre iguales y otros constituye un eje fundamental del derecho internacional ${ }^{32}$.

La racionalidad detrás del principio de igualdad no explica causalmente la actitud de los gobiernos latinoamericanos a principios del siglo XIX. Pero nos permite entender por qué estos gobiernos no hicieron otra cosa que buscar el reconocimiento de los Estados que se reconocían entre ellos como soberanos e iguales. Como veremos ahora, en el siglo XIX, la racionalidad de la igualdad fue implementada a través del estándar de civilización. A partir de la segunda mitad del siglo xx, este estándar evolucionó en dos estándares estrechamente vinculados: desarrollo económico neoliberal y desarrollo institucional del rule of law.

Entendida en el contexto de estas racionalidades, la igualdad parece transformar la soberanía en una simple carta de membrecía de la comunidad internacional. El reconocimiento tanto político como económico siempre parece tener un costo alto en el orden internacional: el costo es volver inalcanzable cualquier aspiración de ser distinto ${ }^{33}$.

\section{a. La aspiración de ser parte de los países civilizados: igualdad y soberanía}

Formar parte del concierto de naciones era para los gobiernos latinoamericanos de principios del siglo XIX un objetivo central ${ }^{34}$. Para ello, la racionalidad de la igualdad requería que los Estados latinoamericanos adoptaran las instituciones europeas consideradas civilizadas. Actuar de esta forma era necesario porque el reconocimiento de quienes ya formaban parte de la comunidad internacional, es decir de los países europeos, era lo que conllevaba la subjetividad internacional. Según la academia internacional de la época, la idea de soberanía y Estado tenía como elementos definitorios la existencia de instituciones civilizadas. Para los nuevos Estados, además, también era importante mostrar que esas instituciones eran naturalmente adecuadas para Latinoamérica. Pretender lo contrario significaba asumir que estas tierras estaban condenadas a la barbarie, a ser el otro $^{35}$.

32 ANGHIE, supra n. 17, p. 4.

33 Esquirol explica que la ortodoxia de derecho internacional latinoamericano intenta alcanzar la soberanía con argumentos de superioridad racial vinculados con la civilización europea. Esquirol, J. Can International Law Help: An Analysis of the Colombian Peace Process. 23 Connecticut Journal of International Law 16, 2000, 80.

34 BECKER, F. Los Tratados de amistad, comercio y navegación y la integración de los estados independientes americanos en el sistema internacional. En Problemas de la formación del Estado y de la nación en Hispanoamérica. 247, 1984.

35 Obregón, L. ¿Para qué un derecho internacional latinoamericano? En René UrueÑa (ed.), Derecho internacional. Poder y límites del derecho en la sociedad global 27. Bogotá: Uniandes, 2014, 37. 
La racionalidad de la igualdad y el estándar de civilización son una racionalidad creada tanto por los tratados internacionales como por la academia de derecho internacional de la época. Esto no es casualidad, si aceptamos que la academia considera que su misión es ordenar las relaciones internacionales. El estándar de civilización tenía un claro elemento constitutivo del orden internacional. En el siglo XIX, este estándar significaba la imposición de principios básicos de la civilización occidental, como la propiedad privada, al igual que el establecimiento de contraprestaciones por los actos de reconocimiento internacional.

La soberanía, es decir, nunca fue gratuita. En el ámbito económico, esto se puede ver en los tratados de paz, amistad y comercio, que muchos gobiernos latinoamericanos firmaron con países europeos como forma de obtener su reconocimiento como Estados. Por un lado, estos tratados establecían una enumeración de estándares de civilización, como la ya mencionada propiedad privada. Por otro lado, determinaban de forma bilateral y recíproca ciertas reglas que respondían a intereses de los países europeos. Un ejemplo es la libre navegación de los ríos. Si bien los dos Estados parte del tratado se beneficiaban de esta cláusula, los nuevo Estados latinoamericanos no tenían flota mercante. Algunos países europeos, en cambio, eran líderes en el transporte marítimo y fluvial. Como algunos autores recalcan, esta regla-que es resultado del principio de igualdad y reciprocidad- limitó las posibilidades de que se creara una flota mercante en Latinoamérica ${ }^{36}$.

Cuando existían grandes divergencias con las instituciones civilizadas europeas, además, eran necesarias concesiones adicionales para obtener el reconocimiento internacional. La deuda contraída por Haití en 1825, por ejemplo, es el resultado de que muchos de sus ciudadanos según los estándares de civilización imperantes (o mejor dicho incivilización) eran esclavos, es decir, propiedad privada de sujetos de otros Estados. Así, Haití tuvo que contraer un empréstito para poder compensar la pérdida de derechos que existían según las leyes vigentes en la metrópoli ${ }^{37}$.

El reconocimiento de los Estados latinoamericanos como soberanos, por otro lado, estaba condicionado a que sus gobiernos actuasen conforme a la racionalidad creada y reproducida por el derecho internacional. Si los Estados latinoamericanos no cumplían con los estándares de civilización fundamentales para el orden internacional, por ejemplo el respeto de la propiedad privada, la comunidad internacional se reservaba su derecho de intervenir para hacerlos valer. En otras palabras, se reservaba el derecho de desconocer el carácter soberano de los nuevos Estados. El principio de no intervención, corolario clave del reconocimiento de soberanía, sólo se aplicaba a los Estados que actuaban de forma civilizada. Esta premisa justificaba la

37 OBREgón, supra n. 35, pp. 32-5. 
protección diplomática en Latinoamérica, y también la institución consular en países como China y Japón ${ }^{38}$.

La idea de soberanía, así, parece más una forma de definir una membrecía a un club que un término para describir la capacidad de un pueblo de gobernarse a sí mismo. La práctica de los nuevos Estados y la posición de sus académicas así lo confirman. En la mayoría de los casos de intervención, los representantes de los nuevos Estados no desafiaban las reglas impuestas por los estándares de civilización, sino que afirmaban que sí cumplían con esos estándares o que estaban en un rápido proceso de implementación de las leyes occidentales ${ }^{39}$. Estas reacciones se pueden explicar de acuerdo al conocimiento dominante de derecho internacional, y demuestran el poder constitutivo de la racionalidad de la igualdad en las relaciones internacionales. Ninguno de los nuevos Estados pretendía ser reconocido en el orden internacional como distinto, o sea, como lo suficientemente autónomo como para darse sus propias instituciones. En el contexto de la racionalidad de la igualdad, en otras palabras, la idea de soberanía es casi antagónica a la idea de autonomía. Una comunidad de personas debía renunciar a su autonomía para formar parte del club de Estados soberanos.

b. La aspiración de ser parte del mundo desarrollado: igualdad, desarrollo y rule of law

Luego del proceso de descolonización se consolida una transformación importante del orden internacional, ya que gran parte del territorio mundial es reconocida como parte integrante de Estados iguales y soberanos. La comunidad internacional de Estados mantiene el control sobre la subjetividad estatal internacional. Gobierno, territorio y población no son más que factores materiales que en la práctica -como en el caso de Palestina- no implican soberanía. Sin embargo, una vez otorgado el reconocimiento, la igualdad y la soberanía formal tienen un carácter menos condicional. Este cambio afecta la racionalidad de la igualdad, haciendo más difícil disciplinar la conducta estatal a partir de la dicotomía civilizado-incivilizado. Pero la influencia sobre los asuntos internos de muchos Estados no desaparece, en la medida que es necesaria para mantener el orden económico global. La academia de DIE realiza esta tarea a partir de otros medios y fines, de otras racionalidades.

Este momento histórico coincide con el surgimiento de una nueva forma de ordenar las relaciones internacionales, que dará al DIE su protagonismo actual. Desde la segunda mitad del siglo xx, el derecho internacional racionaliza gran parte de las relaciones entre los Estados -tanto a nivel regional como

38 BeCKer LorCA, A. Mestizo International Law: a Global Intellectual History 1842-1933. Cambridge: Cambridge University Press, 2014, 3-8.

39 Ibíd. 
global- a partir de la cuestión económica. Se trata más concretamente de la racionalidad del desarrollo económico ${ }^{40}$. Todos los Estados son formalmente iguales, pero no son económicamente iguales. Los Estados que anteriormente eran los administradores de la civilización son ahora los administradores del desarrollo, y el DIE va a reconocer esta diferencia desde el mismo proyecto de Bretton Woods. Por ejemplo, dos de las instituciones clave del orden económico global, el Fondo Monetario Internacional (FMI) y el Banco Mundial, tienen mecanismos de votación que reflejan esta jerarquía o desigualdad.

Como ocurría con el estándar de civilización, existen dos hechos sociales clave que no requieren mayor comprobación en el caso de la racionalidad del desarrollo. Uno es el reconocimiento generalizado de algunos Estados como desarrollados, y el otro es el deseo del resto de alcanzar ese desarrollo. El reconocimiento como desarrollado no se ve alterado por el hecho de que se trate de uno de los países más desiguales del mundo, o de que allí se hayan iniciado las dos crisis financieras más graves de los últimos cien años: nadie cuestiona a los Estados Unidos como un país desarrollado. Por el contrario, existe todo un conocimiento desplegado alrededor de la idea de desarrollo cuyo objetivo es dar forma a la conducta de los países considerados en desarrollo. Este conocimiento económico se manifiesta en la forma de medios y fines, es decir, de racionalidades, y es el resultado del trabajo de académicos y expertos de organismos internacionales económicos, como el Banco Mundial, el FMI y la Organización para la Cooperación y el Desarrollo Económico (OCDE).

Los Estados llamados desarrollados aprueban el deseo de desarrollarse que tienen otros Estados. A veces, podría decirse que lo celebran. Desde finales de la Segunda Guerra Mundial ofrecen ayuda financiera, técnica y política para el desarrollo, que también es funcional a otros intereses: desde luchar contra el comunismo hasta mantener la injerencia en los asuntos internos de otros países $^{41}$. Si bien el resto de los Estados no está obligado a aceptar esta ayuda (o la misma racionalidad del desarrollo), pocos están dispuestos a ser el subdesarrollado, de la misma forma que nadie estaba dispuesto a ser el incivilizado en el siglo xIx. Hay presiones para seguir este camino, como las condicionalidades del FMI, pero no se trata sólo de coerción sino también de la aceptación de ciertos medios como idóneos para alcanzar el desarrollo económico.

40 La importancia de la racionalidad del crecimiento económico en los Estados liberales y neoliberales es desarrollada por Weber y Foucault. Ver Swedberg, R. Max Weber's Contribution to the Economic Sociology of Law. 2 Annual Review of Law and Social Science 61, 2006; Foucault, M. Security, Territory and Population: Lectures at the Collège de France 1977-1978. Chippenham, UK: Palgrave Macmillan, 2009, 95-9, 341-58; Foucault, M. The Birth of Biopolitics: Lectures at the Collège de France, 1978-1979. Chippenham, UK: Palgrave Macmillan, $2010,144,252$.

41 Ver supra n. 25. 
A diferencia de lo que ocurría con la racionalidad de la igualdad y el estándar de civilización, sin embargo, la racionalidad del desarrollo adopta dos formas históricamente diferenciadas. Los Estados del Sur global, al menos durante las primeras décadas de la posguerra, van a cuestionar la racionalidad del desarrollo impulsada por Estados Unidos y Europa occidental. Desconfían porque creen que estos Estados alcanzaron su bienestar material a partir de relaciones asimétricas coloniales o de dominación informal ${ }^{42}$, eliminando, por ejemplo, la posibilidad de una flota mercante en Latinoamérica. Durante las décadas de 1950 a 1970, siguiendo esta línea, los Estados latinoamericanos buscaron desarrollarse mediante una racionalidad propia, inspirada en la teoría de la dependencia.

Impulsada por académicos del Sur global, esta racionalidad del desarrollo se presenta como una demanda, una forma de emancipación, que exige a Estados Unidos y Europa occidental la reorganización de las relaciones económicas internacionales ${ }^{43}$. Se trata de implementar reglas que permitan al resto de los Estados dirigir su economía, principalmente el uso de los recursos naturales, y utilizar esos recursos para alcanzar el desarrollo industrial. Esta faz emancipadora puede verse en el discurso de la teoría de la dependencia sobre comercio e inversiones, que desconfía del libre comercio y del capital proveniente de las antiguas potencias coloniales ${ }^{44}$. Considera, en cambio, que la creación de capital nacional es necesaria para alcanzar tanto el desarrollo económico como una democracia saludable ${ }^{45}$. Se trataba, según Prebisch, de darle a la población de esos Estados las mismas condiciones económicas y democráticas de Estados Unidos y Europa occidental ${ }^{46}$.

Durante este período, la producción académica latinoamericana -y en general de todo el Sur global- resaltaba la importancia de un derecho internacional al desarrollo como base de un nuevo orden económico global ${ }^{47}$. Se trataba de una visión alternativa de las relaciones económicas entre los Estados: de un orden y una racionalidad distintas. Esta visión se plasmó en varias resoluciones de la Asamblea General de la Organización de las Nacio-

42 Gilpin, R. Global Political Economy: Understanding The International Economic Order. Princeton, N.J.: Princeton University Press, 2001, 286-288.

43 Esta demanda se articula a través del derecho al desarrollo. Ver Gross Espiell, supra n. 22 .

44 En el caso latinoamericano, los exponentes más importantes de esta escuela son RAÚL Prebisch, Celso Furtado, Fernando H. Cardozo y Enzo Faletto. Ver Bielschowsky, R. Cincuenta años del pensamiento de la CEPAL: una reseña. En Cincuenta años del pensamiento de la CEPAL: textos seleccionados. Santiago: Fondo de Cultura Económica, CEPAL, 1998, v. 1, 9-61.

45 Cuaderno Sr., M. The Anti-Capitalist Attitude'. En Private investment: The key to international industrial development; a report of the San Francisco Conference. 52, New York: McGraw Hill, 1958; Prebisch, R. El desarrollo económico de la América Latina y algunos de sus principales problemas. 26 Desarrollo Económico 479, 1950, 1986.

46 Ibíd., 479.

47 Gros Espiell, H. supra n. 22, 230-233. 
nes Unidas. Las más importantes se refieren a la soberanía permanente sobre los recursos naturales ${ }^{48} \mathrm{y}$ a la carta de derechos y deberes económicos de los Estados $^{49}$. Algunas de estas ideas se consolidaron -aunque por un período breve- con la creación de la Comisión Económica para América Latina y el Caribe (CEPAL) y de la Conferencia de las Naciones Unidas sobre Comercio y Desarrollo (UNCTAD, por sus siglas en inglés) ${ }^{50}$.

Esta visión emancipadora del desarrollo, no obstante, fue derrotada a finales de la década de 1970 por la racionalidad del desarrollo impulsada por Estados Unidos y Europa occidental. Para esta visión, la clave no era la planeación industrial estatal, sino la atracción de inversión extranjera privada a fin de utilizar de manera eficiente los recursos económicos ${ }^{51}$. Esta racionalidad del desarrollo es neoliberal, y el auge de estas ideas va a circular desde el Norte hacia el Sur ${ }^{52}$. Para alcanzar el desarrollo económico, dice el paradigma neoliberal de la década de 1990, resulta necesario que los Estados se comporten de acuerdo con una nueva disciplina institucional y fiscal. Estas políticas son diseñadas y su implementación monitoreada desde el Banco Mundial, el FMI, la oCDE y la nueva unidad de UNCTAD encargada de inversión extranjera ${ }^{53}$.

En este contexto, los organismos internacionales comenzaron a otorgar financiamiento para implementar las instituciones necesarias para el desarrollo neoliberal. Muchas universidades del Norte apoyarían este proyecto. Así tomó forma la racionalidad de la igualdad y el rule of law, según la cual los países aún no desarrollados deben incorporar las instituciones de Estados Unidos y Europa occidental. Se trata de reconocer derechos de propiedad fuertes, iniciar una reforma del Estado dirigida a privilegiar el rol del mercado como garante del desarrollo económico, y mantener una estabilidad fiscal y macroeconómica ${ }^{54}$. Esta es una racionalidad que busca facilitar la actividad económica global y la atracción de inversión extranjera privada.

En lo que respecta específicamente a las inversiones extranjeras, la racionalidad neoliberal del desarrollo fue consolidada a través de tratados asimétricos de promoción y protección de inversiones. Estos tratados protegen la

48 Resolución 1803 (xVII) de la Asamblea General de la ONU, de 14 de diciembre de 1962.

49 Resolución 3281 (xxIx) de la Asamblea General de la onU, de 12 de diciembre de 1974.

50 Gros EsPiell, supra n. 22, 230-33.

51 Ogle, V. State Rights against Private Capital: The "New International Economic Order" and the Struggle over Aid, Trade, and Foreign Investment, 1962-1981. 5 Humanity 211, 2014.

52 El Banco Mundial, por ejemplo, fue clave en la implementación de reformas agrarias sugeridas por expertos como Hernando de Soto. Ver Esquirol, J. Legal Latin Americanism, 16 Yale Human Rights \& Development Law Journal. 145, 2013, 153-4.

53 En el caso de unctad. Ver Sagafi-Nejad T. y Dunning, J. The UN and Transnational Corporations: from code of conduct to global compact. Bloomington: Indiana University Press, 2008, 124-6.

54 Ver RoBerTs, A. The logic of discipline: global capitalism and the architecture of government. New York: Oxford University Press, 2010. 
inversión de ambos Estados, pero en la práctica la gran mayoría de los flujos provienen de los países del Norte global. Algo no muy distinto de lo que sucedió con los tratados de amistad, navegación y comercio. En el caso del transporte marítimo y fluvial, habría transporte en Latinoamérica pero sería un negocio de extranjeros. En el caso de las inversiones extranjeras hoy, habrá crecimiento económico pero dependerá y será dirigido - principalmente-por capitales globales ${ }^{55}$. Las implicancias de este control extranjero son dejadas de lado o minimizadas por gran parte de la literatura actual sobre arbitraje internacional de inversiones. Este esquema, no obstante, estaría dando lugar al surgimiento de una clase capitalista transnacional que actúa como si fuera externa a las necesidades y valores de cada comunidad ${ }^{56}$.

En la implementación de este orden económico neoliberal, el rol de la Organización Mundial del Comercio (OMC) también fue vital. Este organismo fue durante el final de la década de 1990 el faro de muchas reformas neoliberales en términos comerciales. Su función, al decir de Chang, fue patear la escalera por si acaso algún país pretendía volver a implementar políticas de promoción industrial ${ }^{57}$. Al limitar considerablemente el apoyo a la industria doméstica, incorporar un fuerte sistema de protección de la propiedad intelectual y facilitar la movilidad de bienes y servicios, la OMC consolida el capitalismo internacional de las cadenas globales de valor. Volverse parte de estas cadenas resulta vital en la actual configuración de las relaciones económicas internacionales ${ }^{58}$.

En este contexto de transformación de la década de 1990, gran parte de la academia latinoamericana de DIE se limitó principalmente a seguir los cambios y avances institucionales. Si bien ha habido críticas, como las de Correa en el área de propiedad intelectual ${ }^{59}$, la mayoría de la literatura latinoamericana se ocupó de sistematizar los instrumentos legales, reproduciendo de manera explícita o implícita racionalidades que reproducen el orden económico global neoliberal ${ }^{60}$.

55 Ver LALL, S. Reinventing industrial strategy: the role of government policy in building industrial competitiveness. 28. United Nations Conference on Trade and Development, 2004, 31; ChANG, H. J. Regulation of Foreign Investment in Historical Perspective. 16:3 The European Journal of Development Research. 687, 2004, 712.

56 Ver Chimni, supra n. 5.

57 Ver supra n. 29.

58 Ver Comunicado del G20, 15-16 noviembre de 2015. Disponible en http://www.gpfi. org/publications/g20-leaders-communiqu-antalya-summit-2015 [fecha de consulta: 8 de marzo de 2016].

59 CORREA, C. Intellectual property rights, the WTO and developing countries: the TRIPS agreement and policy options. New York: Zed books, 2000.

60 Rosas, J.E. Derecho internacional económico. Instituciones y críticas contemporáneas. México, Porrúa, 2006, capítulo 2. 
III. LA ACADEMIA LATINOAMERICANA DE DIE Y SU CONTRIBUCIÓN

AL ORDEN ECONÓMICO GLOBAL

La academia latinoamericana de DIE forma parte de la academia internacional. El DIE se presenta hoy al mundo como una disciplina global, a pesar de los tratados bilaterales de libre comercio, del alto grado de fragmentación convencional que existe en el régimen internacional de inversiones, y de los distintos contextos políticos, económicos y sociales de cada país y región. La academia en esta materia no ve estos contextos diferentes como un problema serio, ya que a las divergencias textuales, sociales y políticas se oponen racionalidades globales que sirven para minimizar diferencias y ordenar las relaciones económicas internacionales. Lo verdaderamente global y universal, entonces, es el conocimiento de DIE. La comunidad académica regional involucrada en esta materia no presenta una discontinuidad normativa ni social con la comunidad global: con sólo algunas pocas excepciones, se trata de un subgrupo dentro de esa misma comunidad epistémica ${ }^{61}$. No es casualidad, por ejemplo, que la red latinoamericana de DIE haya sido creada en el seno y sea un capítulo más de la red internacional ${ }^{62}$.

Dada la importancia de Latinoamérica en las relaciones económicas internacionales, supo ser una de las principales voces críticas entre los años 1950 y 1970, los académicos de esta región tienen algún peso en la creación de conocimiento en DIE. Frente a la diversidad de textos legales y a la indeterminación propia del derecho, la existencia de un DIE global muestra que hay un consenso en la creación y reproducción del conocimiento. Gran parte de la academia latinoamericana es hoy parte de ese consenso.

Por un lado, la simple recepción y enseñanza de conocimientos consolida las racionalidades que constituyen y ordenan las relaciones económicas internacionales. Por el otro, las contribuciones que hacen muchos expertos regionales de DIE a los principios y las reglas vigentes consolidan el orden económico global. Estos expertos representan a la región de manera indirecta, y muchos dan su aquiescencia al orden actual. La participación de jueces y árbitros latinoamericanos, muchos de ellos académicos, es un argumento en favor de la legitimidad de un régimen internacional sin importar el contenido de sus decisiones.

\section{a. La contribución a través de la enseñanza}

Como la mayor parte de la enseñanza de derecho en Latinoamérica, la enseñanza del DIE tiene en vista principalmente el mercado profesional, y percibe

61 Estas excepciones se limitan casi exclusivamente al área de propiedad intelectual. Ver supra $n$. 59 y el trabajo de RAFAel Pérez Miranda en el área de biotecnología y semillas.

62 Ver http://iellatina.blogspot.com/ Vale aclarar que el autor es miembro activo de esta red. 
al derecho como una práctica que debe ser aprendida y aplicada a casos concretos $^{63}$. Esta perspectiva deja de lado el rol constitutivo del derecho y el contexto del orden vigente. Si bien esta tendencia es común a todas las áreas del derecho, es más fácil para los estudiantes tener conciencia de que el derecho doméstico, sea de propiedad o de contratos, por ejemplo, es fruto de una puja entre intereses políticos distintos. Esto es así por una cuestión de proximidad social y cultural; el contexto está allí mismo, fuera del aula. En DIE, en cambio, esto es más difícil dada la supuesta lejanía de las relaciones económicas internacionales, que sólo interesarían a las élites y tendrían poco que ver con la vida cotidiana de los trabajadores o los enfermos ${ }^{64}$.

Las competencias internacionales son un ejemplo de la enseñanza de DIE como una práctica ${ }^{65}$. Una de las actividades que más interés despiertan en los estudiantes, en la que más empeño ponen, es la de pensar y resolver disputas, es decir, reparar el orden perdido en una relación económica internacional concreta. En estas competencias, los estudiantes viajan a distintos lugares del mundo para enfrentarse a los expertos que ejecutan diariamente la racionalidad del desarrollo, ya sea de las inversiones extranjeras o del comercio internacional. En el marco de la preparación para estas competencias, la actividad docente consiste en entrenar a los estudiantes a operar dentro de las racionalidades dominantes y del orden establecido. Las competencias internacionales promueven una perspectiva que asume el carácter regulador del DIE, restando importancia al carácter constitutivo que tiene el conocimiento en esta materia. Así, las competencias promueven la formación de nuevos expertos para nutrir a la comunidad que diariamente consolida el orden existente. Por ejemplo, es una práctica de los estudios jurídicos internacionales reclutar "recursos humanos" en estas competencias.

\section{b. La contribución a través de la defensa de los intereses del Estado}

La contribución por medio de la defensa de los intereses estatales es otra forma paradigmática de consolidar el orden existente, en la que participan

63 Ver Lista, C. La construcción de la conciencia jurídica: los objetivos educativos y la formación del abogado, 5 Anuario - CIJS 381, 1999-2000, 394-5, 400; Pérez Perdomo, R. Desafíos de la educación jurídica latinoamericana en tiempos de globalización. 38 El otro derecho 11, 2008, 19-20. La formalización de la enseñanza del derecho como práctica esconde el lado constitutivo del proceso de aprendizaje. Como explica LISTA, no sólo se imparte conocimiento jurídico, sino también se forman conciencias jurídicas: LisTA, ob. cit., 386-7.

64 GERRY SimpSOn reconoce que uno de los problemas de la academia de derecho internacional es la omisión del contexto. Ver supra n. 4, 88.

65 Ver BRAVo, K. International Economic Law in US Law Schools : Evaluating its Pedagogy and Identifying Future Challenges. En PICKER, C. et al. (eds.), International Economic Law: The state and future of the discipline. 135. London: Hart Publishing, 2008, 155. Las competencias internacionales más importantes son la Jessup, la Vis, la FDI Moot y la ELSA-WTO. El autor tiene conocimiento directo de la Vis y la FDI Moot, y conocimiento indirecto de las otras dos. 
académicos y expertos latinoamericanos. En el siglo XIX, por ejemplo, CARLOS Calvo no criticaba la distinción entre países civilizados y no civilizados. Por el contrario, defendía a los Estados latinoamericanos dentro de esta racionalidad, con el argumento de que los mismos respetaban la propiedad privada tal como los Estados europeos o Estados Unidos ${ }^{66}$. Lo mismo hacían los académicos japoneses o chinos, quienes buscaban demostrar que sus países se comportaban conforme la racionalidad imperante, al haber incorporado las instituciones de derecho privado y público de los países occidentales ${ }^{67}$.

Con estos debates y discusiones, los académicos latinoamericanos -como los académicos de otros países no centrales- también influyeron en la construcción del derecho internacional. BECKER LORCA afirma que existió una cierta circularidad o reflexividad entre los expertos de todo el mundo ${ }^{68}$. No obstante, esta circulación de ideas, en mi opinión, debe enmarcarse dentro de las racionalidades de la igualdad y la soberanía, y la dicotomía civilizadoincivilizado. BECKER LORCA describe un tipo de resistencia que hace uso estratégico del conocimiento dominante de derecho internacional. Este uso puede servir para prevalecer en una controversia puntual o doctrinal, pero resulta difícil pensar en un escenario donde pueda modificar las racionalidades dominantes. La participación de académicos latinoamericanos y asiáticos que describe BECKER LORCA puede hasta llegar a describirse como una forma de tragedia, en la que -al final de cuentas- poco cambia en el conocimiento dominante (p. ej., a raíz de la evolución del estándar de civilización al estándar de desarrollo).

Algo parecido ocurre en la actualidad en el DIE. La defensa de los intereses argentinos en los arbitrajes por la crisis del año 2001 fue altamente ineficaz cuando intentó responder con argumentos propios de derecho constitucional argentino, ajenos al DIE $^{69}$. Cuando este país cambió su estrategia, y comenzó a utilizar argumentos de derecho internacional, su posición mejoró y obtuvo algunas victorias en arbitrajes de inversiones. Puede decirse que, incluso, hizo contribuciones importantes al tema de la excepción por estado de necesidad en el marco del orden vigente. Pero no hay dudas de que, al mismo tiempo, ayudó a consolidar el arbitraje de inversiones. Este país, a pesar de la larga lista de condenas en su contra, no ha denunciado sus tratados bilaterales de inversión ni iniciado un proceso de revisión interno.

66 BECKER LORCA, supra n. 18, 302.

67 BECKER LORCA, supra n. 38, 4-5.

68 Ibíd., 7.

69 La posición argentina era conocida como 'doctrina Rosatti'. Ver Rosatti, H. Los tratados bilaterales de inversión. El arbitraje internacional obligatorio y el sistema constitucional argentino. 67:198. La Ley 1, 2003. 
Lo mismo puede argumentarse del rol de Brasil en la omc, que es visto como exitoso por la literatura ${ }^{70}$. Esta opinión se debe, entre otros, a las victorias conseguidas en el área de subsidios agrícolas. Lo que estas victorias muestran es cómo un país como Brasil puede vencer a Estados Unidos o a la Unión Europea con las reglas vigentes. Con esto, la literatura estaría haciendo dos cosas. Por un lado, mostrando las posibilidades que tienen algunos países emergentes en el marco de la omc; algo que es muy valioso, por cierto. Por el otro, legitimando las reglas de la omc frente al resto del mundo.

La contribución al orden económico global hecha por los defensores de los Estados latinoamericanos también es relevante cuando, por sus grandes cualidades, estos individuos son contratados por las firmas globales de abogados. En el ámbito del arbitraje de inversiones, varios abogados prominentes de la región fueron luego contratados por estas firmas. Las relaciones que se establecen entre las defensas de los Estados y estas firmas globales no hacen más que consolidar el orden vigente. Estas relaciones se fortalecen gracias a la rotación de personas desde la defensa de los Estados hacia las firmas globales de abogados.

\section{c. La contribución a través de la investigación}

Los investigadores latinoamericanos en DIE realizan una contribución importante a la consolidación del orden económico global. Esto se debe a motivos académicos y profesionales. Por un lado, hoy muchos de los académicos en la región se forman en el exterior, la lengua dominante en el DIE es el inglés, y muchos latinoamericanos de renombre están fuera de sus países ${ }^{71}$. A esto se le suma la presión de los rankings universitarios y la creciente necesidad de publicar en revistas reconocidas internacionalmente. Por otro lado, en Latinoamérica existe una cercanía entre muchos académicos, la defensa de los Estados y firmas de abogados. Esto es entendible cuando la idea de una carrera académica en derecho es algo aún novedoso en la región.

Sumadas, estas circunstancias no hacen que nuestra academia pierda su connotación latinoamericana. Los académicos en general se ocupan de la situación particular de un país o de la región. Lo que ocurre es que muchos trabajos abordan las problemáticas a partir de las grandes líneas epistemológicas desarrolladas en los centros académicos del Norte. En una palabra, no es que la academia regional olvida la región, sino que se utilizan enfoques producidos en otras latitudes, incorporando así sea de manera implícita las racionalidades que informan esos trabajos ${ }^{72}$.

70 ShafFer, G. et al., The trials of winning at the wTo: what lies behind Brazil's success. 41:2 Cornell International Law Journal 8, 2008.

71 EsQUIROL, supra n. 52, 148-154.

72 Ibíd. 
Esta actitud se explica en un mundo donde el reconocimiento internacional es muy importante para las universidades y sus académicos. Para los que están en Latinoamérica, especialmente, este reconocimiento es valioso si proviene de las universidades del Norte ${ }^{73}$. Las citaciones en las revistas especializadas de los centros académicos más importantes son las más relevantes, es decir, aquellas revistas dirigidas desde fuera de la región. Ser reconocido en estos centros es difícil por sí mismo y, sin dudas, es aún más difícil si un individuo impulsa ideas que se oponen a las racionalidades dominantes.

Así, vemos cómo el conocimiento en DIE no sólo ordena el accionar de los Estados, sino también de los académicos. Esto no es una sorpresa ya que críticas agudas y profundas ponen en riesgo el orden de las relaciones económicas internacionales. El problema para los que quieren promover el cambio desde la academia es que para cambiar es necesario sugerir alguna forma de crisis en el paradigma de DIE vigente ${ }^{74}$.

Un ejemplo del papel que juega la academia regional puede encontrarse en el derecho de inversiones extranjeras, probablemente el área más controvertida de DIE en la actualidad. Latinoamérica es la región más demandada por inversores extranjeros, y algunos de sus gobiernos han sido muy duros con el arbitraje de inversiones. Sin embargo, no es la región desde donde se han hecho las principales contribuciones académicas críticas. Mi punto aquí no es que la academia regional no ha criticado esta área del DIE. Pero si miramos el contexto global, las críticas más fuertes provienen de académicos canadienses y europeos. Los primeros respondieron duramente luego de que su país fuera condenado en más de una oportunidad en el marco del Tratado de Libre Comercio de América del Norte ${ }^{75}$. Un grupo de académicos europeos, más recientemente, se ha manifestado públicamente en contra del arbitraje de inversiones en el marco de las negociaciones transatlánticas ${ }^{76}$.

En Latinoamérica, en cambio, las críticas gubernamentales no han ido acompañadas de argumentos de DIE novedosos, ni de un apoyo mayoritario

73 OBREGÓN, supra n. 35, 51.

74 Kunn, T. The essential tension: Selected studies in scientific tradition and change. Chicago: University of Chicago Press, 1977.

75 Ver VAn Harten, G. Investment treaty arbitration and public law. New York: Oxford University Press, 2007; SchneIderman, D. Constitutionalizing Economic Globalization. Investment Rules and Democracy's Promise. New York: Cambridge University Press, 2008. Ver, también, la declaración de Osgoode impulsada por VAN HARTEN y SCHNEIDERMAn, donde sólo dos de quienes suscriben tienen puestos académicos o de investigación en Latinoamérica. Disponible en http://www.osgoode.yorku.ca/public-statement-international-investment-regime-31-august-2010/ [fecha de consulta: 8 de marzo de 2016].

76 Statement of Concern about Planned Provisions on Investment Protection and InvestorState Dispute Settlement (ISDS) in the Transatlantic Trade and Investment Partnership (TTIP), University of Kent. Disponible en https://www.kent.ac.uk/law/isds_treaty_consultation.html [fecha de consulta: 8 de marzo de 2016]. 
de la academia regional. Se trata, en una palabra, de una crítica moderada ${ }^{77}$. Esta tendencia se vincula tanto a las racionalidades vigentes, es decir a la necesidad de alcanzar el desarrollo, como a las dinámicas académicas y profesionales que dominan la región.

\section{CONCLUSIONES}

El objetivo de este trabajo ha sido inspirar un debate sobre el rol de la academia latinoamericana de DIE. Desde una perspectiva doctrinal dirigida a la práctica, la respuesta es que la labor de la academia es analizar las reglas y servir de apoyo para las negociaciones internacionales que avanzan frenéticamente. El objetivo es brindar a los Estados latinoamericanos los mejores resultados posibles en el actual orden económico global. Esta postura nos dirá que los Estados y las empresas latinoamericanos necesitan abogados que defiendan sus intereses aquí y ahora, y que la academia tienen entonces un rol formador y de apoyo muy importante.

Esta visión explica por qué muchos Estados de la región se encuentran embarcados en la negociación de tratados de libre comercio, mientras otros -que se sienten rezagados en esa carrera-intentan sortear algunos obstáculos institucionales para poder sumarse ${ }^{78}$. Su actitud es razonable si asumimos que estos tratados son la nueva normalidad, y que por lo tanto cuanto más se demore un país en sumarse probablemente más costoso le resultará hacerlo en el futuro ${ }^{79}$. Pero cualquiera sea la opinión que uno tenga sobre los tratados de libre comercio, no es necesario aceptar que esta realidad es ineludible. La academia latinoamericana de DIE puede hacer más que aprender a manejar reglas: también puede trabajar para transformar esta realidad.

Los intereses en juego son demasiado importantes para contentarse con una actitud pasiva. Urge que los académicos trabajen por la defensa de los intereses concretos que se dirimen en una disputa, pero también que la academia sume sus esfuerzos por un DIE que permita a los Estados latinoamericanos implementar políticas que respondan a sus necesidades. Se trata de enseñar no sólo para la asesoría privada o pública, sino también para contar con ciudadanos responsables en el contexto local y global ${ }^{80}$. La región necesita que

77 BECKER LORCA sostiene que la academia de derecho internacional latinoamericano se moderó luego de la década de 1970; ver BECKER LORCA, supra n. 18, 288.

78 Esta es la situación actual de Brasil y Uruguay, que no pueden negociar tratados de libre comercio por las limitaciones que les impone el Mercosur. Ver http://www.lanacion.com. ar /1814925-hacia-un-nuevo-mercosur [fecha de consulta: 8 de marzo de 2016]; http://g1 .globo. com/economia/noticia/2015/05/uruguai-esta-de-acordo-com-brasil-sobre-tratado-entre-mercosule-ue.html [fecha de consulta: 8 de marzo de 2016].

79 Kolsky Lewis, M.The Trans-pacific Partnership Agreement and Development. En Voon, T. (ed.). Trade Liberalisation and International Co-operation. A Legal Analysis of the Trans-Pacific Partnership Agreement. Cheltenham, UK: Edward Elgar, 2013, 28-49.

80 Simpson, G. supra n. 4, 84. 
los académicos sean conscientes del rol constitutivo que tiene la academia en el orden económico global. De lo contrario, las alternativas de política van a estar siempre limitadas a sumarse a la nueva coalición global de mercados abiertos, en palabras de ROBERT ZoELlick, o al ostracismo económico ${ }^{81}$.

Mantener abierta la posibilidad de pensar alternativas, es cierto, no es tarea fácil. La academia latinoamericana de DIE tiene la posibilidad de ser más consciente de su rol constitutivo del orden económico global. Pero estas posiciones, a veces consideradas más críticas, se encuentran a menudo frente a objeciones doctrinales o a la exigencia de soluciones concretas. Sobre lo primero, es esperable que el conocimiento dominante de DIE objete las visiones alternativas del orden económico global bajo el pretexto de que son políticas y parciales. Sin embargo, como queda claro en este trabajo, lo mismo puede decirse del conocimiento dominante. Respecto de los pedidos de soluciones concretas, estos parten de una aceptación implícita del orden vigente. Estos pedidos no admiten que una solución verdadera puede requerir cambios en los términos del debate o en la forma de pensar los problemas.

Pensar alternativas, en otras palabras, requiere una actitud activa frente a la creación de conocimiento. Pero la articulación de alternativas concretas, además, se enfrenta a un obstáculo epistemológico. El DIE actual sólo puede ser entendido a partir de ciertas premisas neoclásicas, neoliberales y postneoliberales que dan forma al orden vigente. Por ejemplo, la relación entre la inversión extranjera y el libre comercio con el desarrollo es clave. Esta conexión está siempre presente en la racionalidad actual del desarrollo. El DIE podía tener un contenido emancipador para el Sur global cuando existía una visión económica alternativa del comercio y las inversiones: la teoría de la dependencia y de la integración económica regional brindaban este apoyo epistemológico. En la actualidad, la falta de una visión económica alternativa hace difícil para los académicos de DIE articular una visión del orden económico vigente donde se tengan en cuenta las desigualdades tanto materiales como normativas.

Al mismo tiempo, también es necesario tener presentes las limitaciones epistemológicas impuestas por el propio DIE. Esta área del conocimiento está ineludiblemente ligada a la globalización económica. Para quienes la globalización es un problema, entonces, es probable que una respuesta satisfactoria se encuentre en discursos más radicales que los que se pueden articular desde el DIE. De la misma forma, ciertos aportes -p. ej., desde la sociología y la antropología- pueden ser muy importantes para repensar el DIE, pero también deben ser utilizados con cuidado si lo que se intenta es reordenar las relaciones económicas internacionales. El riesgo que se corre es que algunos esfuerzos sean recibidos como voces exóticas por los académicos de $\mathrm{DIE}^{82}$.

81 Zoellick, R. Unleashing the trade winds. The Economist, 5 December 2002.

82 El trabajo de Boaventura De SouZa Santos sobre epistemologías del Sur, por ejemplo, 
Los inversores extranjeros, por ejemplo, actúan por el beneficio esperado y no por caridad. Cualquier regulación de la inversión extranjera deberá tener presente esta premisa, a menos que su objetivo sea terminar con la inversión extranjera y la globalización. No corresponde abrir aquí un debate sobre la viabilidad de estas opciones más radicales, sino simplemente notar que el DIE puede ser un espacio de oportunidades para quienes creen que un día cada país y región podrá escoger su destino en la globalización económica.

\section{BIBLIOGRAFÍA}

Аввott, Kenneth y Duncan Snidal. Why states act through formal international organizations. 42:1 Journal of Conflict Resolution 3, 1998.

Anghie, Anthony. Imperialism, Sovereignty and the Making of International Law. New York: Cambridge University Press, 2004.

BAADE, HANs. Teaching International Economic Law. 16 Journal of Legal Education 59, 1963.

Becker Lorca, Arnulf. International Law in Latin America or Latin American International Law-Rise, Fall, and Retrieval of a Tradition of Legal Thinking and Political Imagination. 47 Harvard International Law Journal 283, 2006.

Becker Lorca, Arnulf. Mestizo International Law: A Global Intellectual History 18421933. Cambridge: Cambridge University Press, 2014.

BeCKer, Félix. Los Tratados de amistad, comercio y navegación y la integración de los Estados independientes americanos en el sistema internacional. En Problemas de la formación del Estado y de la Nación en Hispanoamérica 247, 1984.

Bedjaoui, Mohammed. Towards a new international economic order. New York: Holmes \& Meier, 1979.

Bielschowsky, Ricardo. Cincuenta años del pensamiento de la CEPAL: una reseña. En Cincuenta años del pensamiento de la CEPAL: Textos seleccionados 9. Santiago: Fondo de Cultura Económica-CEPAL, 1998.

Bravo, Karen. International Economic Law in US Law Schools: Evaluating its Pedagogy and Identifying Future Challenges. En Colin PICKer et al. (eds.), International Economic Law: The State and Future of the Discipline 135. London: Hart Publishing, 2008.

Chang, Ha-Joon. Regulation of Foreign Investment in Historical Perspective. 16:3 The European Journal of Development Research 687, 2004.

Chang, Ha-Joon. Kicking away the Ladder: Development Strategy in Historical Perspective. London: Anthem, 2002.

es una herramienta importante para repensar el DIE. Sin embargo, puede presentar el riesgo de transformar un discurso alternativo en una voz exótica. En este sentido, ver EsQuirol, supra $\mathrm{n}$. 52,166 . 
Chimni, Bhupinder. International Institutions Today: An Imperial Global State in the Making. 15:1 European Journal of International Law 1, 2004.

Correa, Carlos. Intellectual Property Rights, the wTo and Developing Countries: The TRIPS Agreement and Policy Options. New York: Zed Books, 2000.

Cuaderno Sr., Miguel. The Anti-Capitalist Attitude. En Private Investment: The Key to International Industrial Development. A Report of the San Francisco Conference 52. New York: McGraw Hill, 1958.

EnRíQuez Rosas, José. Derecho internacional económico. Instituciones y críticas contemporáneas, México, Porrúa, 2006.

Esquirol, Jorge. Can International Law Help: An Analysis of the Colombian Peace Process, 23 Connecticut Journal of International Law 16, 2000.

Esquirol, Jorge. Legal Latin Americanism. 16 Yale Human Rights \& Development Law Journal 145, 2013.

FERRER, Aldo. La importancia de las ideas propias sobre el desarrollo y la globalización. 173: 44 Revista Problemas del Desarrollo 163, 2013.

Foucault, Michel. Technologies of the self, en P. Rabinov (ed.), Ethics: Subjectivity and Truth, New York: The New York Press, 1997.

Foucault, Michel. Security, Territory and Population: Lectures at the Collège de France 1977-1978, Chippenham, UK: Palgrave Macmillan, 2009.

Foucault, Michel. Society must be defended: Lectures at the Collège de France, 19751976, London: Penguin, 2004.

Foucault, Michel, The Birth of Biopolitics: Lectures at the Collège de France, 1978-1979, Chippenham, UK: Palgrave Macmillan, 2010.

Gilpin, Robert. Global Political Economy: Understanding the International Economic Order, Princeton, N.J.: Princeton University Press, 2001.

Gross Espiell, Héctor. Derecho internacional del desarrollo, en Estudios de Derecho Económico II, 1. ${ }^{\text {a }}$ reimp., México: Universidad Autónoma de México, 1980.

Kolsky Lewis, Meredith. The Trans-Pacific Partnership Agreement and Development, en Tania Voon (ed.), Trade Liberalisation and International Co-operation. A Legal Analysis of the Trans-Pacific Partnership Agreement 28, Cheltenham, UK: Edward Elgar, 2013.

Koskenniemi, Martti. The Politics of International Law, 1 European Journal of International Law 4, 1990.

Kunn, Thомаs. The essential tension: Selected studies in scientific tradition and change, Chicago: University of Chicago Press, 1977.

LaLl, SANJAYA. Reinventing industrial strategy: The role of government policy in building industrial competitiveness. En United Nations Conference on Trade and Development, 2004. 
LANG, ANDREw. Governing 'As If': Global Subsidies Regulation and the Benchmark Problem, 67, 1 Current Legal Problems 135, 2014.

LANG, ANDREW. World Trade Law after neoliberalism: Re-imagining the global economic order, Oxford: Oxford University Press, 2011.

Lista, Carlos. La construcción de la conciencia jurídica: los objetivos educativos y la formación del abogado, 5 Anuario - CIJS 381, 1999-2000.

Obregón, Liliana. ¿Para qué un derecho internacional latinoamericano? En René Urueña (ed.), Derecho internacional. Poder y límites del derecho en la sociedad global 27, Bogotá: Uniandes, 2014.

Ogle, Vanessa. State Rights against Private Capital: The "New International Economic Order" and the Struggle over Aid, Trade, and Foreign Investment, 1962-1981. $5 \mathrm{Hu}$ manity 211, 2014.

Pahuja, Sundhya. Decolonising International Law: Development, economic growth, and the politics of universality. Cambridge: Cambridge University Press, 2011.

PAPARINSKIS, Martins. The international minimum standard and fair and equitable treatment. Oxford: Oxford University Press, 2013.

PAulsson, JAN. Arbitration without privity. 10:2 ICSID Review 232, 1995.

Pérez Perdomo, Rogelio. Desafíos de la educación jurídica latinoamericana en tiempos de globalización. 38 El Otro Derecho 11, 2008.

Perrone, Nicolás. The International Investment Regime and Foreign Investors' Rights: Another View of a Popular Story. PhD Thesis, London School of Economics and Political Science, 2013.

Petersmann, Ernst-Ulrich. Transformation of the World Trading System through the 1994 Agreement Establishing the World Trade Organization. 6 European Journal of International Law 161, 1995.

Prebisch, Raúl. El desarrollo económico de la América Latina y algunos de sus principales problemas, 26 Desarrollo Económico 479, 19501986.

Rasulov, Aквar. The Structure of the International Legal Discourse, European Society of International Law, Florence Founding Conference, 2004.

Roberts, Alasdair. The logic of discipline: Global capitalism and the architecture of government, New York: Oxford University Press, 2010.

Rodrik, Dani. The Global Governance of Trade - As if Development Really Mattered, United Nations Development Programme Background Paper, 2001.

Rosatti, Horacio. Los tratados bilaterales de inversión. El arbitraje internacional obligatorio y el sistema constitucional argentino, 67: 198 La Ley 1, 2003.

Ruggie, John. What makes the world hang together? Neo-utilitarianism and the social constructivist challenge 52:4 International Organization 855, 1998. 
Sagafi-Nejad, Tagi y John Dunning, The UN and Transnational Corporations: from code of conduct to global compact, Bloomington: Indiana University Press, 2008.

Schneiderman, David. Constitutionalizing Economic Globalization. Investment Rules and Democracy's Promise, New York: Cambridge University Press, 2008.

Serra, Narcís; Shari Spiegel y Joseph Stiglitz, Introduction: From the Washington Consensus Towards a New Global Governance. En The Washington Consensus Reconsidered: Towards a New Global Governance 3, New York : Oxford University Press, 2008.

Shaffer, Gregory et al., The trials of winning at the wTo: What lies behind Brazil's success, 41:2 Cornell International Law Journal 8, 2008.

Simpson, Gerry. On the magic mountain: Teaching public international law, 10:1 European Journal of International Law 70, 1999.

Swedberg, Richard. Max Weber's Contribution to the Economic Sociology of Law, 2 Annual Review of Law and Social Science 61, 2006.

Trebilcock, Michael y Robert Howse. The regulation of international trade, New York: Routledge, 1999.

VAn Harten, Gus. Investment treaty arbitration and public law, New York: Oxford University Press, 2007.

WADE, RoBert. What strategies are viable for developing countries today? The World Trade Organization and the shrinking of 'development space', 10:4 Review of International Political Economy 621, 2003.

Weber, Max. El sentido de la 'neutralidad valorativa' de las ciencias sociológicas y económicas en Ensayos sobre Metodología Sociológica, Buenos Aires: Amorrortu, 1973.

Weber, Max. Parliament and Government in Germany under a New Political Order, en P. Lassman (ed.), Weber: Political Writings, Cambridge: Cambridge University Press, 1994.

Zoellick, Robert. Unleashing the trade winds, The Economist, 5 December 2002. 